\title{
Amentoflavone Induces Apoptosis and Reduces Expression of Anti-apoptotic and Metastasis-associated Proteins in Bladder Cancer
}

\author{
CHIH-HUNG CHIANG ${ }^{1,2,3}$, CHING-YI YEH $^{4}$, JING GUNG CHUNG $^{5,6}$, I-TSANG CHIANG $^{7,8,9}$ and FEI-TING HSU ${ }^{5}$ \\ ${ }^{1}$ Department of Urology, Medical Research and Education, \\ Taipei Veterans General Hospital, Yuan-Shan/Su-Ao Branch, Yilan, Taiwan, R.O.C.; \\ ${ }^{2}$ Department of Nursing, Cardinal Tien Junior College of Healthcare and Management, New Taipei, Taiwan, R.O.C.; \\ ${ }^{3}$ Department of Urology, National Taiwan University Hospital, Taipei, Taiwan, R.O.C.; \\ ${ }^{4}$ Department of Nursing, Taipei Veteran General Hospital, Yuan-Shan/Su-Ao Branch, Yilan, Taiwan, R.O.C.; \\ ${ }^{5}$ Department of Biological Science and Technology, China Medical University, Taichung, Taiwan, R.O.C.; \\ ${ }^{6}$ Department of Biotechnology, Asia University, Taichung, Taiwan, R.O.C.; \\ ${ }^{7}$ Department of Radiation Oncology, Show Chwan Memorial Hospital, Changhua, Taiwan, R.O.C.; \\ ${ }^{8}$ Department of Medical Imaging and Radiological Sciences, \\ Central Taiwan University of Science and Technology, Taichung, Taiwan, R.O.C.; \\ ${ }^{9}$ Department of Radiation Oncology, Chang Bing Show Chwan Memorial Hospital, Changhua, Taiwan, R.O.C.
}

\begin{abstract}
Background/Aim: Amentoflavone has been shown to be effective against a variety of cancer cells, but its role in bladder cancer remains unclear. Thus, the aim of this study is to evaluate whether amentoflavone may induce toxicity effect of bladder cancer. Materials and Methods: Herein, we evaluated amentoflavone effects in a human bladder cancer cell line TSGH8301 in vitro. Results: Amentoflavone caused significant cytotoxicity in TSGH8301 cells at a concentration as low as $200 \mu M$. FAS/FASL-dependent extrinsic apoptosis and mitochondria-dependent intrinsic apoptosis were observed in amentoflavone-treated cells in a dose-dependent manner. Levels of several proapoptotic proteins, such as FAS, $F A S$-ligand and BAX (B-cell lymphoma 2 associated $X)$ were increased following amentoflavone treatment. Meanwhile, anti-apoptotic MCL-1 (myeloid cell leukemia sequence 1) and cellular FLICE-inhibitory protein (C-FLIP) protein levels were reduced. Additionally, angiogenesis and proliferationrelated proteins, including matrix metalloproteinase (MMP)2, -9, vascular endothelial growth factor (VEGF), urokinase-
\end{abstract}

Correspondence to: Fei-Ting Hsu, Department of Biological Science and Technology, China Medical University, Taichung, Taiwan 404, R.O.C. Tel: +886 926302759, e-mail: sakiro920@gmail.com and ITsang Chiang, Department of Radiation Oncology, Chang Bing Show Chwan Memorial Hospital, Changhua 505, Taiwan, R.O.C. Tel: +886 983035179, e-mail: john740604@gmail.com

Key Words: Amentoflavone, extrinsic apoptosis, intrinsic apoptosis, anti-apoptotic, metastasis. type plasminogen actvator (uPA) and cyclin DI were diminished by amentoflavone. Conclusion: Amentoflavone induced toxicity of bladder cancer by inhibiting tumor progression and inducing apoptosis signaling transduction.

Many carcinogens lead to bladder cancer through genetic alterations (1). The molecules that cause genetic alterations promote the overexpression of oncogenes and the silencing of tumor suppressor genes, leading to apoptosis dysregulation and metastasis in bladder cancer (2-4). Overexpression of anti-apoptotic and metastasis-associated proteins often observed in high-grade and invasive bladder tumors inhibit the therapeutic efficacy of current treatment approaches for bladder cancer, including radical cystectomy, chemotherapy, and radiotherapy (5-7). Anti-apoptotic and metastasis-associated proteins are recognized as potential targets for the treatment of bladder cancer $(8,9)$.

Herbal medicine, including compounds and formulas derived from natural plants, are applied to treat and prevent urinary tract diseases, such as urinary stone disease and urinary tract infections $(10,11)$. In addition, herbal medicines have been demonstrated to possess anticancer properties in bladder cancer. Lee et al., presented their work on herbal medicines and have shown that they can effectively reduce the number and size of multiple metastatic nodules in both lungs in a 74-year-old Korean man (12). Flavonoids, bioactive compounds extracted from plants, such as curcumin and apigenin, are indicated to inhibit cell invasion and induce apoptosis in bladder cancer $(13,14)$ Zamora-Ros et al., have found that dietary intake of 
flavonoid subclasses (flavonol) and lignans are associated with a reduction in bladder cancer risk (15).

Amentoflavone, a bioflavonoid isolated from Selaginella tamariscina, has been indicated to modulate the activity of several cancer types. Amentoflavone inhibits metastatic activity by suppressing the activation of nuclear factor-kappaB (NF-kB) in melanoma, osteosarcoma, and breast cancer (1618). Amentoflavone can also induce apoptosis and reduce the expression of anti-apoptotic proteins in glioblastoma and breast cancer $(19,20)$. In a previous study, we found amentoflavone enhances the therapeutic efficacy of sorafenib, the oral multikinase inhibitor, by inducing apoptosis in HCC (21). However, whether amentoflavone promotes apoptosis and abolishes the expression of anti-apoptotic and metastasisassociated proteins in bladder cancer is ambiguous. The aim of the present study was to investigate the anticancer properties of amentoflavone in bladder cancer in vitro.

\section{Materials and Methods}

Chemical reagents and antibodies. Amentoflavone, 3-(4,5Dimethylthiazol-2-yl)-2,5-diphenyltetrazolium bromide (MTT) and dimethyl sulfoxide (DMSO) were purchased from Sigma Chemical Co. (St. Louis, Missouri, USA). Primary antibodies used in Western blots were acquired from different companies, such as XIAP (Thermo Fisher Scientific, Fremont, California, USA), C-FLIP (Cell signaling Technology, Danvers, Massachusetts, USA), MCL-1 (BioVision, Milpitas, California, USA), BAX (Proteintech Inc., Rosemont, Illinois, USA), FAS (Elabscience Biotechnology Inc., Houston, Texas, USA), FASL (Elabscience Biotechnology Inc.), VEGF (EMD Millipore, Billerica, MA, USA), MMP-2 (Proteintech Inc.), MMP-9 (EMD Millipore), uPA (abcam, Cambridge, MA, USA), Cyclin D1 (Thermo Fisher Scientific), $\beta$-actin (Santa Cruz, California, USA). All secondary antibodies were purchased from Jackson ImmunoResearch (West Grove, Pennsylvania, USA).

TSGH8301 cells culture. Human bladder carcinoma cell line (TSGH 8301) was kindly provided from Professor Jing-Gung Chung's lab, China Medical University $(22,23)$. TSGH 8301 cells were cultured in $10 \mathrm{~cm}$ culture plates and were preserved in flasks in a humidified atmosphere of $5 \% \mathrm{CO}_{2}$ at $37^{\circ} \mathrm{C}$. The culture medium of TSGH 8301 contained RPMI-1640 medium, $10 \%$ heat inactivated fetal bovine serum (FBS), $2 \mathrm{mM} \mathrm{L-glutamine,} \mathrm{and} 1 \%$ antibiotics (100 units $/ \mathrm{ml}$ penicillin and $100 \mu \mathrm{g} / \mathrm{ml}$ streptomycin). Cell culture reagents were all obtained from GIBCO $^{\circledR} /$ Invitrogen Life Technologies (Carlsbad, California, USA).

Cell viability analysis. TSGH 8301 cells were seeded into 96-well plates with a density of $2 \times 10^{4}$ cells/well overnight. Cells were then treated with $0-300 \mu \mathrm{M}$ amentoflavone for 24 or $48 \mathrm{~h}$, respectively. Cell viability was performed using the MTT assay, as previous described (1). The absorbance of MTT was detected using SpectraMax iD3 at $570 \mathrm{~nm}$ wavelength (Molecular Devices, San Jose, California, USA).

Annexin-V/PI flow cytometry analysis. TSGH 8301 cells were seeded into 6-well plates with a density of $5 \times 10^{5}$ cells/well overnight. Cells were then treated with $0,150,200 \mu \mathrm{M}$ amentoflavone for $48 \mathrm{~h}$. Cells were finally collected and double

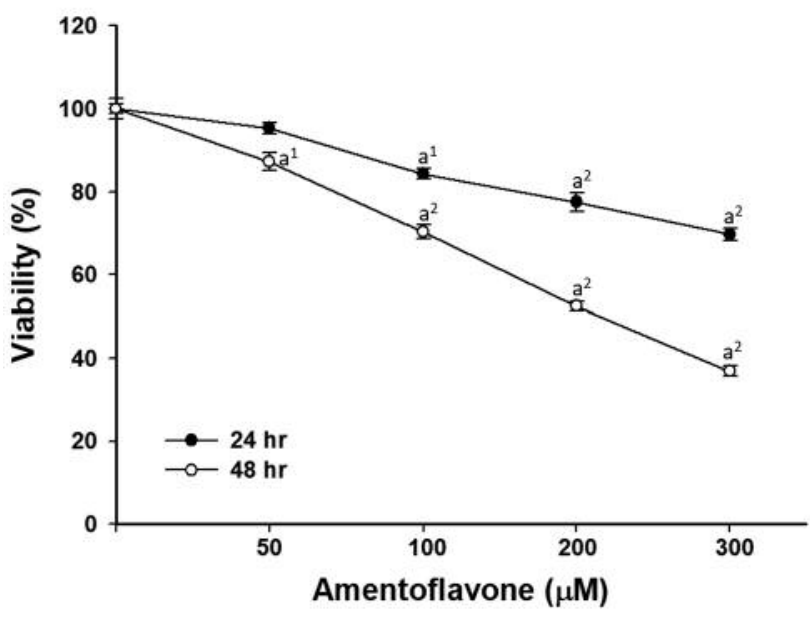

Figure 1. Cell viability was markedly decreased by amentoflavone. MTT assay was used to validate the viability of TSGH 8301 cells following amentoflavone treatment. $\left(a^{1} p<0.05\right.$ and $a^{2} p<0.01$ vs. $0 \mu M$ amentoflavone).

stained using Annexin V/PI in the dark for 15 min (24). Detection and quantification of the positive Annexin V-FITC/PI-PE population was performed using FACSCalibur flow cytometry (BectonDickinson, Franklin Lakes, New Jersey, USA) and FlowJo software, version 7.6.1 (FlowJo LLC, Ashland, Oregon, USA), respectively. Annexin V-FITC apoptosis detection kit was purchased from Vazyme Biotech Co. Lt (Nanjing City, PR China).

Cleaved caspase- 3 and caspase-8 flow cytometry analysis. TSGH 8301 cells were seeded into 6 -well plates with a density of $5 \times 10^{5}$ cells/well overnight. Cells were then treated with $0,150,200 \mu \mathrm{M}$ amentoflavone for $48 \mathrm{~h}$. Cells were finally collected and stained using the CaspGlow fluorescein active Caspase- 3 and -8 staining kit (BioVision). The detailed staining procedure has been described in our previous study (24). The percentages of cleaved caspase- 3 and caspase- 8 were detected and quantified using FACSCalibur flow cytometry and FlowJo software, respectively.

Cell-cycle flow cytometry analysis. TSGH 8301 cells were seeded into 6 -well plates with a density of $5 \times 10^{5}$ cells/well overnight. Cells were then treated with $0,150,200 \mu \mathrm{M}$ amentoflavone for $48 \mathrm{~h}$. For cell cycle phase analysis, cells were harvested, fixed in $70 \%$ ethanol and stored at $-20^{\circ} \mathrm{C}$ overnight. The second day, cells were centrifuged and stained with propidium iodide (PI: $40 \mu \mathrm{g} / \mathrm{ml}$ ) at $25^{\circ} \mathrm{C}$ for $60 \mathrm{~min}$ (24) (Sigma Chemical Co., St. Louis, MO, USA). Detection and quantification of the sub-G1 cell population was performed using FACSCalibur flow cytometry and FlowJo software, respectively. PI and RNase were purchased from purchased from Sigma-Aldrich and Fermentas (St. Leon-Rot, Baden-Württemberg, Germany), respectively (25).

FAS/FASL flow cytometry analysis. TSGH 8301 cells $\left(5 \times 10^{5}\right.$ cells/well) were seeded into 6-well plates overnight before treatment. Cells were then treated with $0,150,200 \mu \mathrm{M}$ amentoflavone. After a 48-hour treatment, cells were harvested and double stained usingut anti-FAS-FITC and anti-FASL-PE for $15 \mathrm{~min}$ on ice, purchased from Thermo Fisher Scientific $(1,24)$. Detection and quantification of the FAS and FASL percentage was assayed by 
FACSCalibur flow cytometry and FlowJo software, respectively. Mitochondria membrane potential flow cytometry analysis. Half a million of TSGH 8301 cells were seeded into 6-well plates overnight. Cells were then treated with $0,150,200 \mu \mathrm{M}$ amentoflavone. Following a 48-hour treatment, cells were collected and stained by $4 \mu \mathrm{M} \mathrm{DiOC}$ in $500 \mu \mathrm{l}$ PBS for 30 minutes at $37^{\circ} \mathrm{C}$. The loss of mitochondria membrane potential was assayed using FACSCalibur flow cytometry and was quantified by FlowJo software. DiOC $_{6}$ was purchased from Enzo Life Sciences (Farmingdale, New York, NY, USA) $(1,24,25)$.

Western blot analysis. Three million TSGH 8301 cells were incubated in $10 \mathrm{~cm}$ culture plates overnight and then treated with i) 0 , ii) 150 , iii) or $200 \mu \mathrm{M}$ amentoflavone. Total cells were collected and re-suspended in lysis buffer $[50 \mathrm{mM}$ Tris- $\mathrm{HCl}(\mathrm{pH}$ $8.0), 120 \mathrm{mM} \mathrm{NaCl}, 0.5 \%$ Nonide P-40] for sonication. Following centrifugation at $13,000 \mathrm{rpm}$, total proteins from supernatant were measured using the Pierce BCA protein assay kit (Thermo Fisher Scientific) with bovine serum albumin (BSA) as the standard. Proteins were then separated by $8-12 \%$ of sodium dodecyl sulfate polyacrylamide gel electrophoresis (SDS-PAGE) and were transferred onto polyvinylidene difluoride (PVDF) membrane (EMD Millipore). Membranes were then processed with blocking (5\% low-fat milk), followed by primary and secondary antibody staining, as described previously (1). Finally, membranes were visualized using enhanced chemiluminescence solution (EMD Millipore) and the chemiluminescent image system (ChemiDoc-It 515, UVP, Upland, CA, USA). VisionWorks LS Analysis Software (UVP) was used to quantify changes in protein expression by densitometry analysis and using $\beta$-actin as the loading control.

Statistical analysis. Statistical significance was measured by Student's $t$-test analysis. $p$-Values $<0.05$ and $<0.01$ were both considered statistically significant. The statistical analysis was conducted using the Excel 2017 software (Microsoft, Redmond, WA, USA). All experiments were repeated independently three times.

\section{Results}

Amentoflavone induced cytotoxicity of TSGH 8301 bladder cancer cells. Following incubation with various concentrations of amentoflavone $(0,50,100,200$ and $300 \mu \mathrm{M})$ for 24 and 48 $\mathrm{h}$, MTT assay was performed in TSGH 8301 cells to measure their viability. Figure 1 results indicated that cell viability decreased in a time- and dose-dependent manner compared to the untreated control. The half maximal inhibitory concentration $\left(\mathrm{IC}_{50}\right)$ of amentoflavone on TSGH 8301 was defined as $200 \mu \mathrm{M}$ treatment for $48 \mathrm{~h}$.

Amentoflavone induced apoptosis and suppressed antiapoptosis effect of TSGH 8301 bladder cancer cells. TSGH 8301 cells treated with 150 and $200 \mu \mathrm{M}$ amentoflavone for 48 $\mathrm{h}$ contained a significantly higher percentage of AnnexinV/PI positive late apoptotic cells compared to the untreated control group (Figure 2A). In Figure 2B, the cleaved form of caspase3 was markedly increased about $30-50 \%$ by amentoflavone as compared to the untreated control group. Moreover, cells in the sub-G1 phase are also recognized as apoptotic; therefore, treatment with amentoflavone induced apoptosis in TSGH 8301 cells (Figure 2C). Additionally, the expression of XIAP, an inhibitor of caspase-3, was markedly decreased by amentoflavone (Figure 2D). In the amentoflavone-treated group, further mitochondria-related anti-apoptosis molecules, such as C-FLIP and MCL-1 were also decreased by $50-80 \%$ as compared to the control group.

Amentoflavone boost apoptosis effect through the induction of extrinsic apoptosis mechanism. TSGH 8301 cells with distinct doses of amentoflavone were evaluated for an extrinsic apoptosis effect. As showed in Figures 3A and B, death receptor FAS and death receptor ligand FASL was effectively increased by amentoflavone treatment. Furthermore, the downstream apoptosis mechanism of FAS/FASL contained caspase- 8 molecule. As shown in Figure 3C, amentoflavone significantly boosted the activation of cleaved caspase-8. The protein expression of BAX, FAS and FASL following amentoflavone treatment showed obviously enhancement as compared to untreated control (Figures 3D and E). Taken together, amentoflavone can successfully trigger FAS/FASL-dependent extrinsic apoptosis signaling in bladder cancer cells.

Amentoflavone triggered intrinsic apoptosis mechanism through the modulation of mitochondria membrane potential $(\Delta \Psi \mathrm{m})$. TSGH 8301 cells were treated with 150 and $200 \mu \mathrm{M}$ amentoflavone for $48 \mathrm{~h}$. The level of $\Delta \Psi \mathrm{m}$ was analyzed and quantified using flow cytometry. Levels of $\Delta \Psi \mathrm{m}$ loss were significantly increased in TSGH 8301 cells treated with amentoflavone (Figure 4) in a dosedependent manner. These results indicate that amentoflavone can induce mitochondria-dependent intrinsic apoptosis signaling.

Amentoflavone diminished angiogenesis, metastasis and proliferation effect in TSGH 8301 bladder cancer cells. For further investigating whether amentoflavone suppressed cell angiogenesis, metastasis and proliferation we assessed the expression of proteins related to tumor progression in TSGH 8301 cells. Following incubation with amentoflavone $(0,150$ and $200 \mu \mathrm{M}$ ) for $48 \mathrm{~h}$, cells were harvested for Western blotting (Figures 5A and B). The results revealed several key angiogenesis-related and metastasis-related proteins, such as VEGF, MMP-2, MMP-9, uPA, which all underwent a significant reduction following 48-hour treatment with amentoflavone. Additionally, protein levels of the proliferation-related molecule, cyclin D1, were also reduced about $50-80 \%$ with amentoflavone. In sum, amentoflavone successfully suppressed the expression of tumor progression related proteins in TSGH 8301 bladder cancer cells, with a profound anti-cancer effect. 


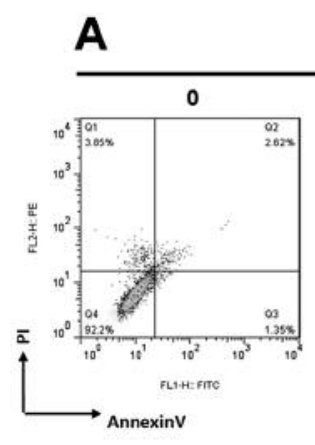

Amentoflavone $(\mu \mathrm{M})$
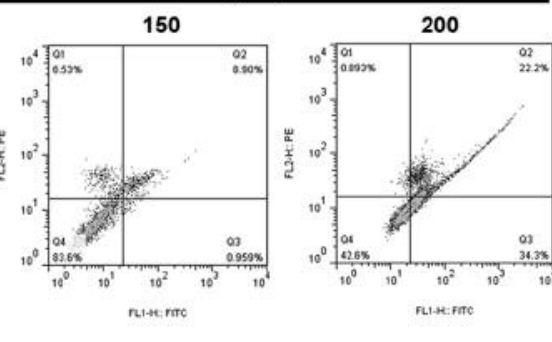

B

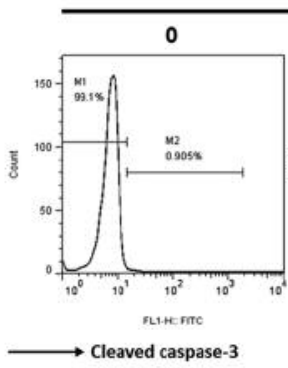

Amentoflavone $(\mu \mathrm{M})$

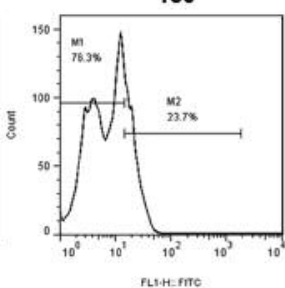

PL:H:Am

$\longrightarrow$ Cleaved caspase-3

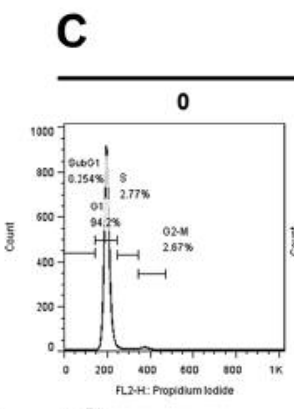

Amentoflavone $(\mu \mathrm{M})$
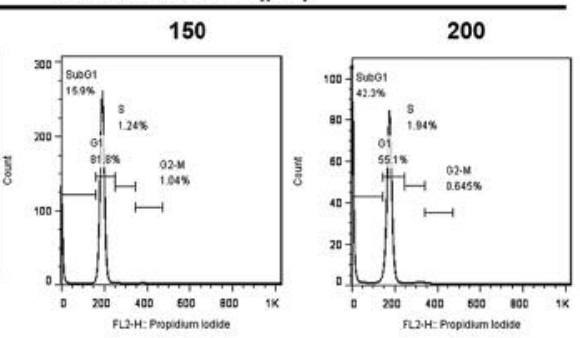

$\rightarrow \mathrm{PI}$

D

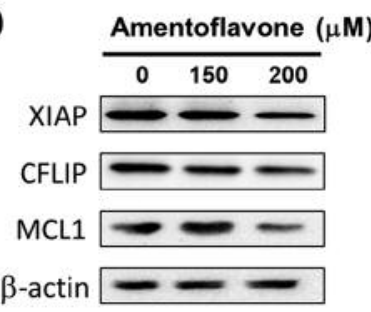

E

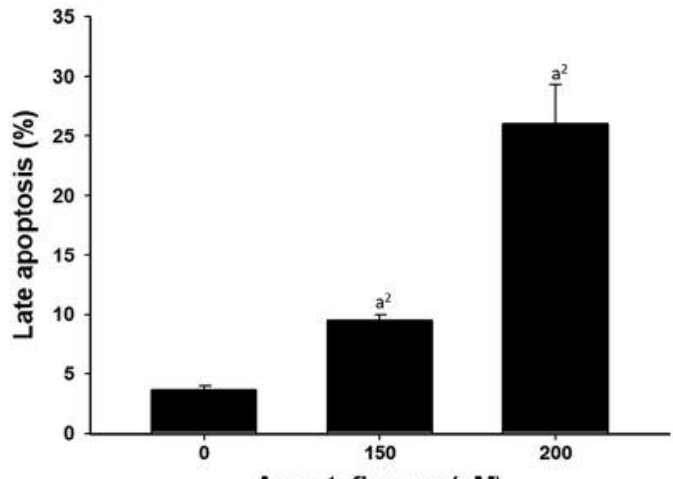

Amentoflavone $(\mu \mathrm{M})$
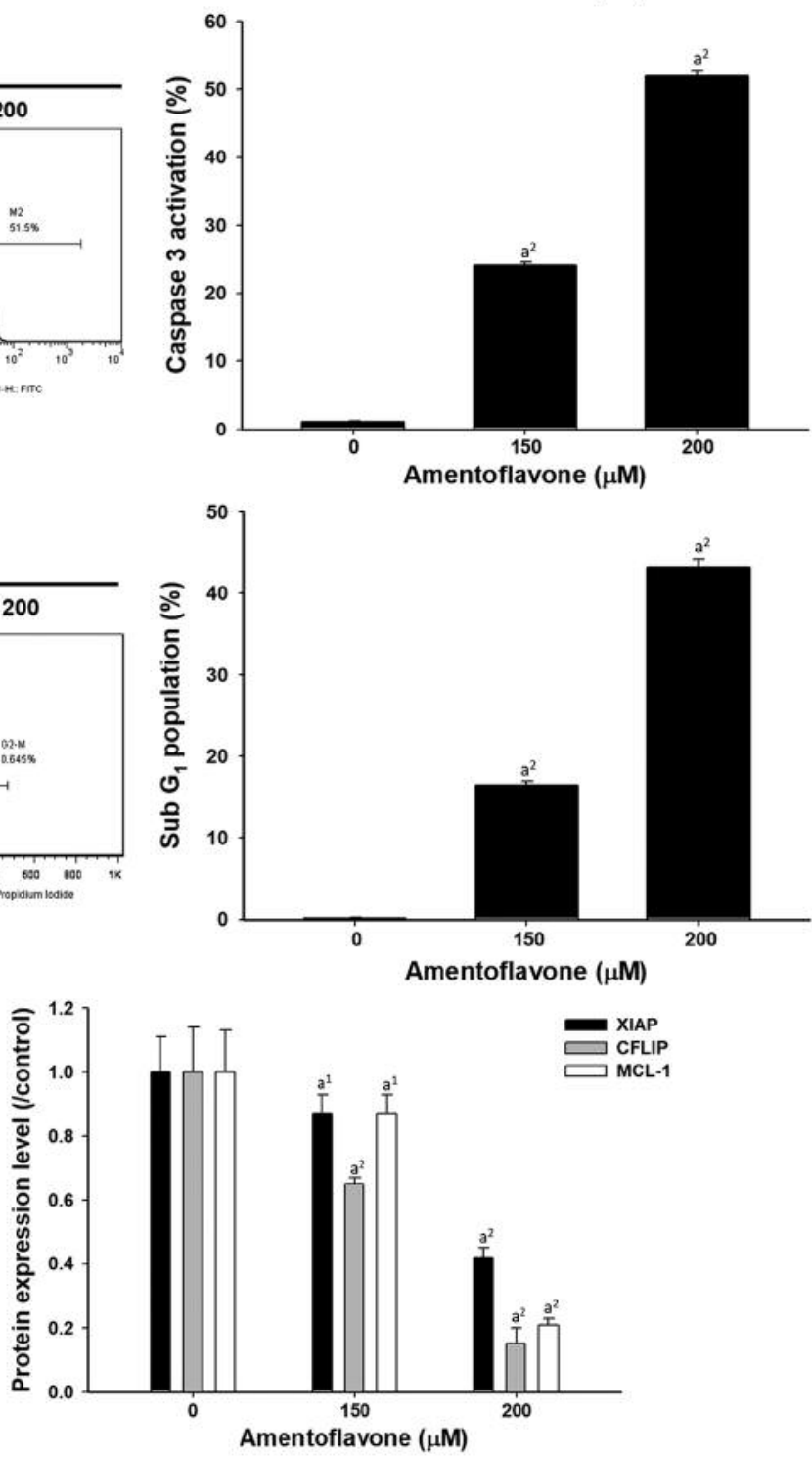

Figure 2. Apoptosis effect was enhanced and anti-apoptosis-related protein expression was reduced by amentoflavone. TSGH 8301 cells were treated with 150 and $200 \mu M$ amentoflavone for $48 \mathrm{~h}$ and were stained with (A) Annexin-V/PI (B) cleaved caspase-3 (C) cell cycle-PI and were analyzed by flow cytometry. Histogram images of each group and quantification results of three repeated experiments are displayed (left and right panels, respectively). (D) Representative chemiluminescence images of XIAP, C-FLIP and MCL-1. (E) Quantification results of the protein expression of XIAP, C-FLIP and MCL-1. ( $a^{1} p<0.05$ and $a^{2} p<0.01$ vs. $0 \mu M$ amentoflavone $)$. 

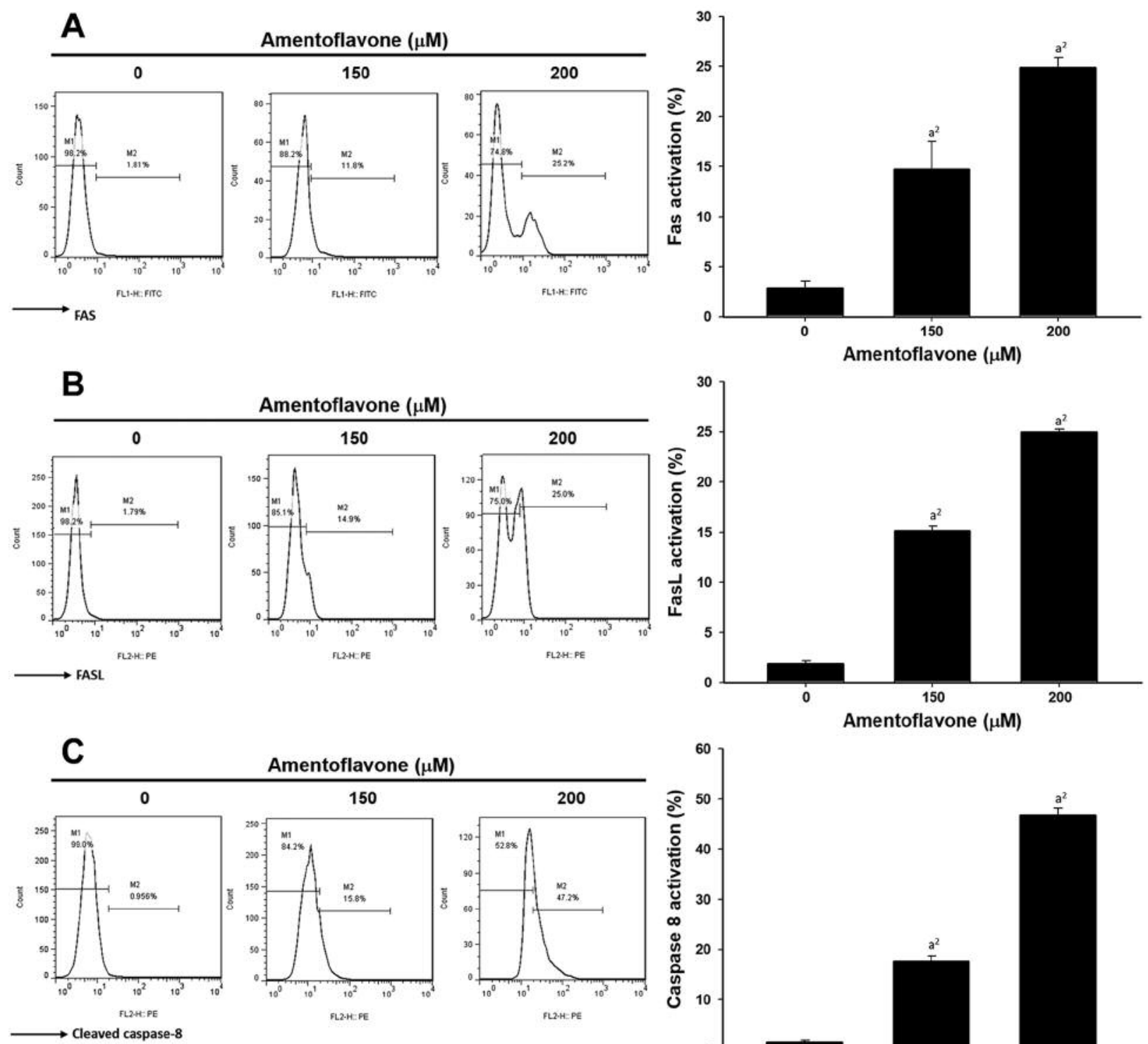

Amentoflavone $(\mu \mathrm{M})$
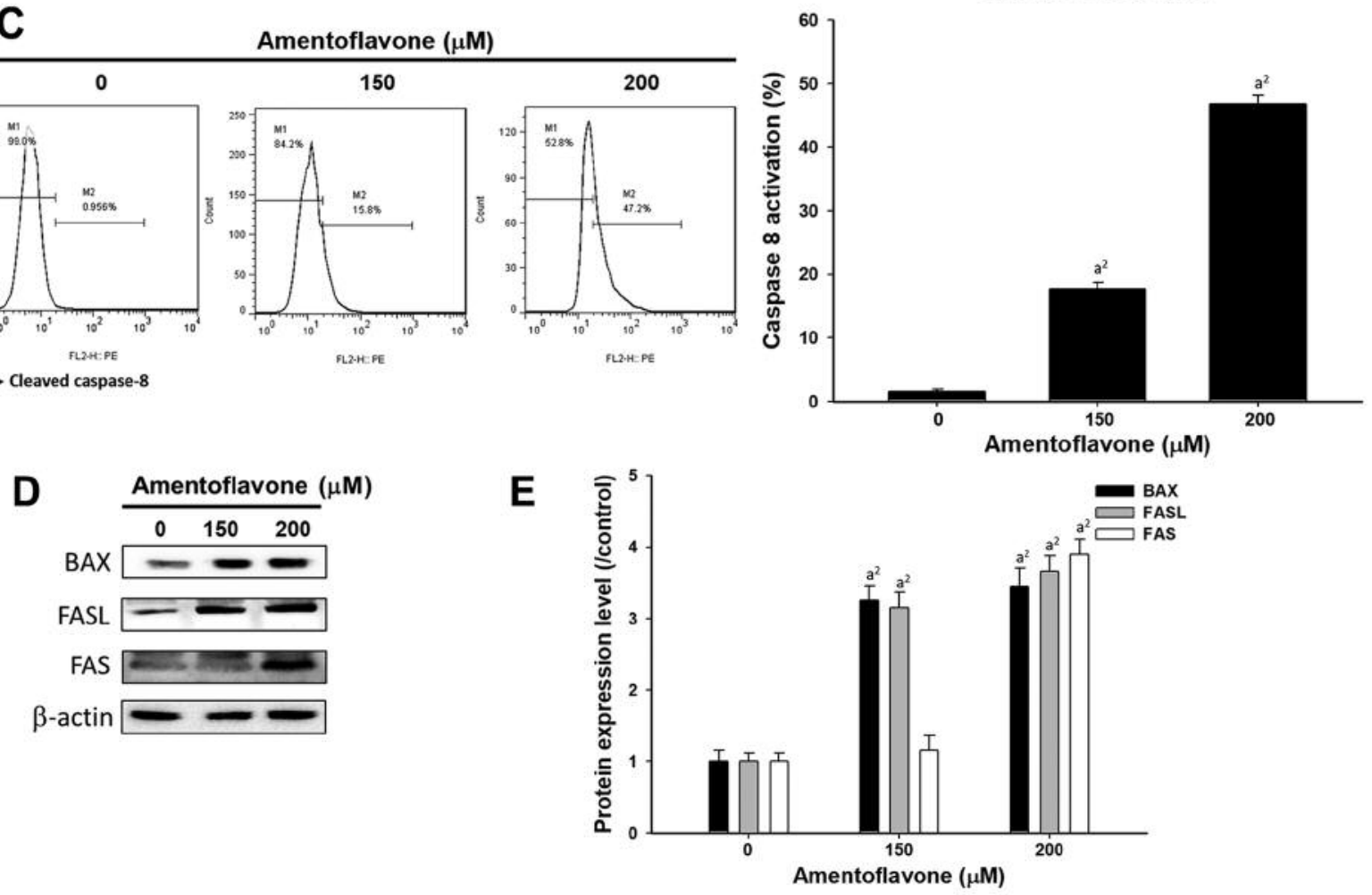

Figure 3. Extrinsic apoptosis signaling was induced by amentoflavone. TSGH 8301 cells were treated with 150 and $200 \mu \mathrm{M}$ amentoflavone for $48 \mathrm{~h}$. Cells were then harvested and stained with (A) FAS (B) FASL (C) cleaved caspase-8 and analyzed by flow cytometry. Histogram images of each group and quantification results of three repeated experiment are displayed. (D) Representative chemiluminescence images of BAX, FAS and FASL. (E) Quantification results of the protein expression of BAX, FAS and FASL. ( $a^{2} p<0.01$ vs. $0 \mu M$ amentoflavone). 

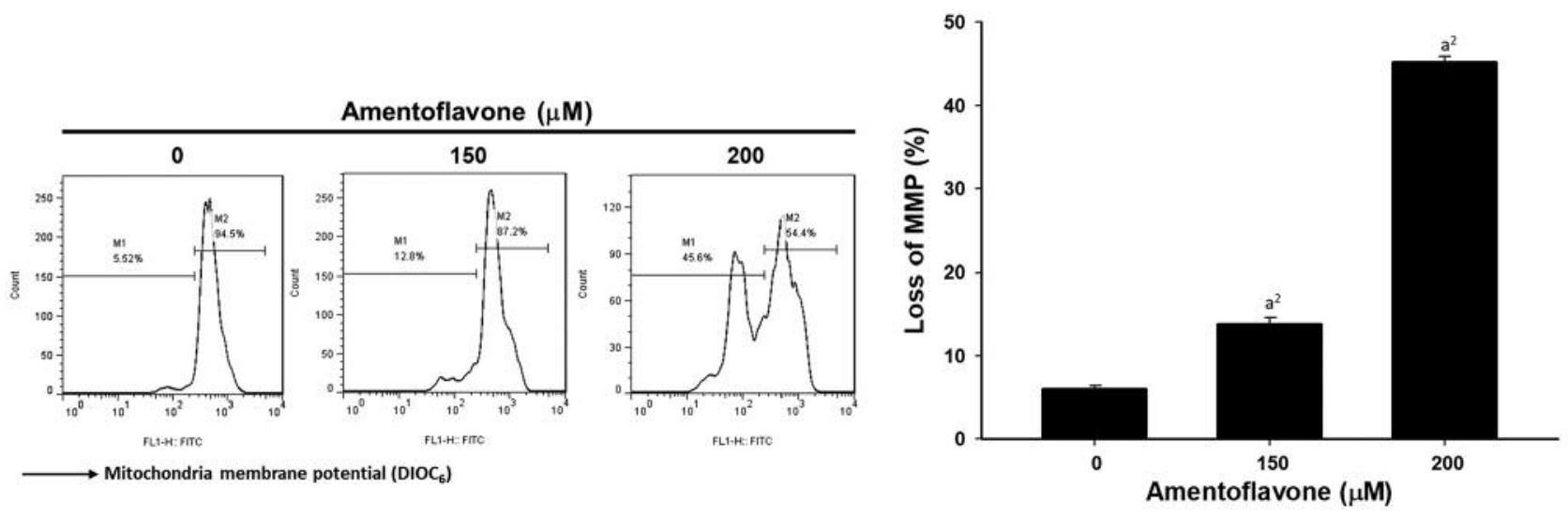

Figure 4. Intrinsic apoptosis signaling was induced by amentoflavone. TSGH 8301 cells were treated with 150 and $200 \mu \mathrm{M}$ amentoflavone for $48 \mathrm{~h}$ and were assesed for $\Delta \Psi_{m}$ by flow cytometry. Histogram images of each group and quantification results of three repeated experiment were displayed. ( $a^{2} p<0.01$ vs. 0 MM amentoflavone).
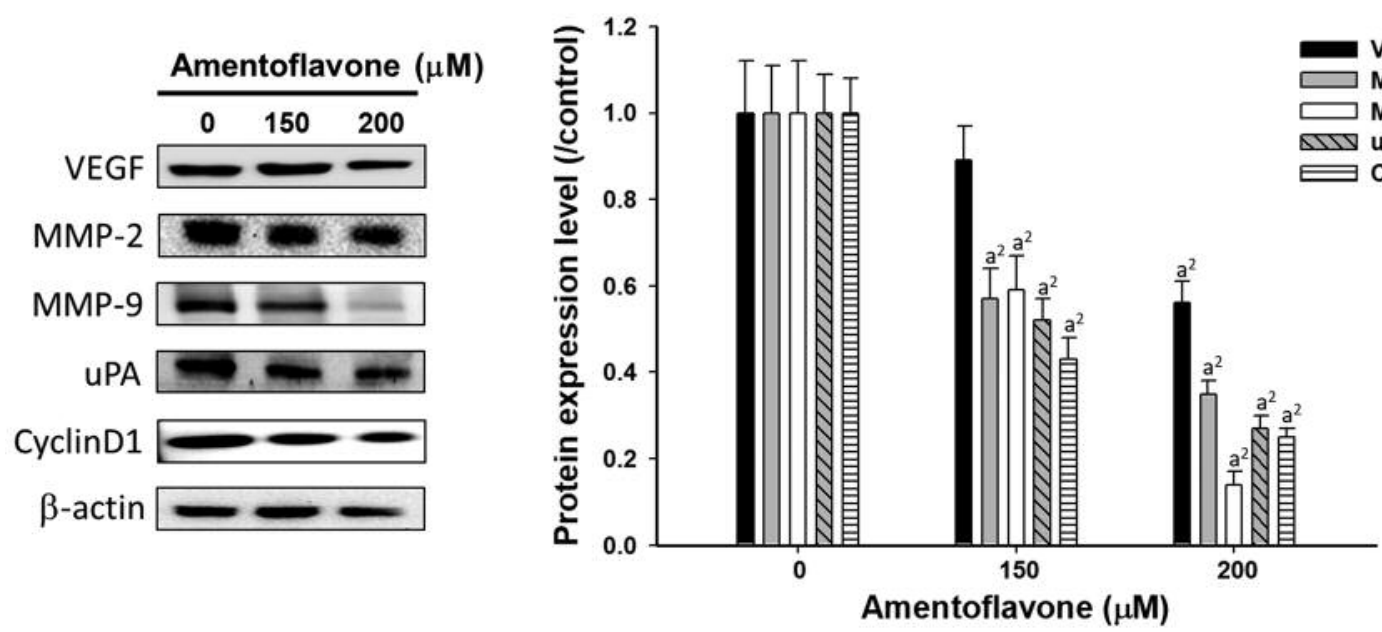

Figure 5. Proliferation and angiogenesis-related protein expression were both decreased by amentoflavone. TSGH 8301 cells were treated with 150 and $200 \mu \mathrm{M}$ amentoflavone for $48 \mathrm{~h}$ and were collected for western blotting analysis. (A) Representative chemiluminescence images of various proteins. (B) Protein level quantification results of three independent experiments. ( $a^{2} p<0.01 \mathrm{vs.} 0 \mu \mathrm{M}$ amentoflavone).

\section{Discussion}

Dysregulation of apoptosis is associated with absence or inactivation of pro-apoptotic proteins and overexpression of anti-apoptotic proteins. Evasion of apoptosis is required for disease progression and development of treatment resistance in cancer (26). The function of several apoptotic proteins is modulated or diminished in bladder cancer (6). The death receptor FAS/FAS ligand (FAS-L) interaction is recognized as the initiator for the induction of the apoptosis extrinsic pathway (27). Bax, the pro-apoptotic member of the B-cell lymphoma 2 (Bcl-2) family, promotes the apoptosis intrinsic pathway by inducing loss of mitochondrial membrane potential $(\Delta \Psi \mathrm{m})$ (28). Both extrinsic and intrinsic apoptotic signaling pathways activate caspase-3, which is essential for apoptotic DNA fragmentation (29). Lack of FAS, Bax, or caspase-3 expression has been shown to correlate with poor prognosis in patients with bladder cancer (30-32).

Several anti-apoptotic proteins have been shown to mediate resistance to apoptosis by blocking the extrinsic and intrinsic apoptotic pathways (33). Overexpression of XIAP (the inhibitor of caspase-3 activation), C-FLIP (the inhibitor of caspase- 8 activation), and MCL-1 (the inhibitor of BAX function at mitochondria) have all been correlated with poor prognosis in patients with bladder cancer (34-36). Inhibition of MCL-1, C-FLIP, or XIAP expression can downregulate cell survival and enhance the antitumoral efficacy of 
chemotherapeutic agents in bladder cancer (36-38). Our results here showed that amentoflavone significantly induces cytotoxicity and apoptosis in bladder cancer cells. Amentoflavone increased the expression of apoptotic proteins, FAS, FAS-L, and BAX, and diminished the expression of XIAP, MCL-1, and C-FLIP. Furthermore, extrinsic and intrinsic apoptotic signaling pathways, including FAS, FAS-L, caspase-8 activation, and loss of $\Delta \Psi \mathrm{m}$ were significantly increased by amentoflavone treatment in TSGH-8301.

Overexpression of metastasis-associated proteins contributes to tumor metastasis, which is the leading cause of death in cancer patients $(17,19)$. Both MMP-2 and MMP-9 have been shown to mediate cancer invasion and metastasis through the disruption of extracellular matrix (ECM) (39). Tumor cells attract blood vessels through the secretion of VEGF and the formation of new blood vessels that supports tumor growth and metastasis (40). The serine protease, uPA, promotes tumor invasion and metastasis by inducing the conversion of plasminogen to plasmin that also participates in ECM degradation (41). Overexpression of uPA, MMP-2, MMP-9, and VEGF has been associated with a poor outcome in patients with bladder cancer (41-43). Our results also demonstrated that protein levels of VEGF, MMP-2, MMP-9, and UPA were significantly inhibited when TSGH-8301 cells were treated with amentoflavone.

In conclusion, amentoflavone not only induces apoptosis through extrinsic and intrinsic pathways, but also inhibits the expression of anti-apoptotic and metastasis-associated proteins in TSGH-8301 cells. We suggest that amentoflavone may be used as a potential adjuvant to provide therapeutic efficacy for the treatment of bladder cancer.

\section{Conflicts of Interest}

The Authors declare that they have no conflicts of interest for this article.

\section{Authors' Contribution}

$\mathrm{CC}$, IC and $\mathrm{FH}$ designed, performed the experiment and analyzed the data, CY and IC supervised the study, JC CC, IC and FH wrote the original manuscript and reviewed and edited the final version.

\section{Acknowledgements}

This study was supported by a grant from the Ministry of Science and Technology, Taipei, Taiwan (grant number: MOST 107-2314B-039-068-MY2). This work was supported by Taipei Veterans General Hospital, Yuan-Shan Branch (grant numbers: YSVH10805 and YSVH10806). This work was also financially supported by the "Drug Development Center, China Medical University" from The Featured Areas Research Center Program within the framework of the Higher Education Sprout Project by the Ministry of Education (MOE) in Taiwan.

\section{References}

1 Chiang $\mathrm{CH}$, Chung JG and Hsu FT: Regorefenib induces extrinsic/intrinsic apoptosis and inhibits mapk/nf-kappabmodulated tumor progression in bladder cancer in vitro and in vivo. Environ Toxicol 34(6): 679-688, 2019. PMID: 30801954. DOI: $10.1002 /$ tox. 22734

2 Comprehensive molecular characterization of urothelial bladder carcinoma. Nature 507(7492): 315-322, 2014. PMID: 24476821. DOI: $10.1038 /$ nature 12965

3 Hovey RM, Chu L, Balazs M, DeVries S, Moore D, Sauter G, Carroll PR and Waldman FM: Genetic alterations in primary bladder cancers and their metastases. Cancer Res 58(16): 35553560, 1998. PMID: 9721860.

4 Ahmad I, Sansom OJ and Leung HY: Exploring molecular genetics of bladder cancer: Lessons learned from mouse models. Dis Model Mech 5(3): 323-332, 2012. PMID: 22422829. DOI: 10.1242/dmm.008888

5 Karam JA, Lotan Y, Karakiewicz PI, Ashfaq R, Sagalowsky AI, Roehrborn CG and Shariat SF: Use of combined apoptosis biomarkers for prediction of bladder cancer recurrence and mortality after radical cystectomy. Lancet Oncol 8(2): 128-136, 2007. PMID: 17267327. DOI: 10.1016/S1470-2045(07)70002-5

6 McKnight JJ, Gray SB, O'Kane HF, Johnston SR and Williamson KE: Apoptosis and chemotherapy for bladder cancer. J Urol 173(3): 683-690, 2005. PMID: 15711244. DOI: 10.1097/01.ju.0000143194.79287.a9

7 Offersen BV, Knap MM, Horsman MR, Verheijen J, Hanemaaijer $\mathrm{R}$ and Overgaard J: Matrix metalloproteinase- 9 measured in urine from bladder cancer patients is an independent prognostic marker of poor survival. Acta Oncol 49(8): 1283-1287, 2010. PMID: 20843171. DOI: 10.3109/0284186X.2010.509109

8 Fassan M, Trabulsi EJ, Gomella LG and Baffa R: Targeted therapies in the management of metastatic bladder cancer. Biologics 1(4): 393-406, 2007. PMID: 19707309.

9 Zeng FC, Cen S, Tang ZY and Kang XL: Elevated matrix metalloproteinase- 9 expression may contribute to the pathogenesis of bladder cancer. Oncology letters 11(3): 2213-2222, 2016. PMID: 26998151. DOI: 10.3892/ol.2016.4187

10 Kasote DM, Jagtap SD, Thapa D, Khyade MS and Russell WR: Herbal remedies for urinary stones used in india and china: A review. J Ethnopharmacol 203: 55-68, 2017. PMID: 28344029. DOI: $10.1016 /$ j.jep.2017.03.038

11 Zhang C, Chen MJ and Tong ZQ: Efficacy of chinese herbal medicine in a case of geriatric urinary tract infections with pain of episiotomy scar inflammation. Medicine (Baltimore) 97(52): e13695, 2018. PMID: 30593139. DOI: 10.1097/MD.000000 0000013695

12 Lee DH, Kim SS, Seong S, Woo CR and Han JB: A case of metastatic bladder cancer in both lungs treated with korean medicine therapy alone. Case Rep Oncol 7(2): 534-540, 2014. PMID: 25232323. DOI: 10.1159/000365884

13 Shi J, Zhang X, Shi T and Li H: Antitumor effects of curcumin in human bladder cancer in vitro. Oncol Lett 14(1): 1157-1161, 2017. PMID: 28693289. DOI: 10.3892/ol.2017.6205

14 Zhu Y, Mao Y, Chen H, Lin Y, Hu Z, Wu J, Xu X, Xu X, Qin J and Xie L: Apigenin promotes apoptosis, inhibits invasion and induces cell cycle arrest of t24 human bladder cancer cells. Cancer Cell Int 13(1): 54, 2013. PMID: 23724790. DOI: $10.1186 / 1475-2867-13-54$ 
15 Zamora-Ros R, Sacerdote C, Ricceri F, Weiderpass E, Roswall N, Buckland G, St-Jules DE, Overvad K, Kyro C, Fagherazzi G, Kvaskoff M, Severi G, Chang-Claude J, Kaaks R, Nothlings U, Trichopoulou A, Naska A, Trichopoulos D, Palli D, Grioni S, Mattiello A, Tumino R, Gram IT, Engeset D, Huerta JM, Molina-Montes E, Arguelles M, Amiano P, Ardanaz E, Ericson U, Lindkvist B, Nilsson LM, Kiemeney LA, Ros M, Bueno-deMesquita HB, Peeters PH, Khaw KT, Wareham NJ, Knaze V, Romieu I, Scalbert A, Brennan P, Wark P, Vineis P, Riboli E and Gonzalez CA: Flavonoid and lignan intake in relation to bladder cancer risk in the european prospective investigation into cancer and nutrition (epic) study. Br J Cancer 111(9): 1870-1880, 2014 PMID: 25121955. DOI: 10.1038/bjc.2014.459

16 Guruvayoorappan C and Kuttan G: Effect of amentoflavone on the inhibition of pulmonary metastasis induced by b16f-10 melanoma cells in c57bl/6 mice. Integr Cancer Ther 6(2): 185197, 2007. PMID: 17548797. DOI: 10.1177/1534735407302345

17 Pan PJ, Tsai JJ and Liu YC: Amentoflavone inhibits metastatic potential through suppression of erk/nf-kappab activation in osteosarcoma u2os cells. Anticancer Res 37(9): 4911-4918, 2017. PMID: 28870912. DOI: 10.21873 /anticanres.11900

18 Chen JH, Chen WL and Liu YC: Amentoflavone induces antiangiogenic and anti-metastatic effects through suppression of nfkappab activation in mcf-7 cells. Anticancer Res 35(12): 66856693, 2015. PMID: 26637885.

19 Yen TH, Hsieh CL, Liu TT, Huang CS, Chen YC, Chuang YC, Lin SS and Hsu FT: Amentoflavone induces apoptosis and inhibits nf-kb-modulated anti-apoptotic signaling in glioblastoma cells. In Vivo 32(2): 279-285, 2018. PMID: 29475910. DOI: 10.21873 /invivo. 11235

20 Pei JS, Liu CC, Hsu YN, Lin LL, Wang SC, Chung JG, Bau DT and Lin SS: Amentoflavone induces cell-cycle arrest and apoptosis in mcf-7 human breast cancer cells via mitochondriadependent pathway. In Vivo 26(6): 963-970, 2012. PMID: 23160679

21 Tsai JJ, Hsu FT, Pan PJ, Chen CW and Kuo YC: Amentoflavone enhances the therapeutic efficacy of sorafenib by inhibiting antiapoptotic potential and potentiating apoptosis in hepatocellular carcinoma in vivo. Anticancer Res 38(4): 2119-2125, 2018. PMID: 29599330. DOI: 10.21873 /anticanres.12452

22 Ho CC, Huang AC, Yu CS, Lien JC, Wu SH, Huang YP, Huang HY, Kuo JH, Liao WY, Yang JS, Chen PY and Chung JG: Ellagic acid induces apoptosis in tsgh8301 human bladder cancer cells through the endoplasmic reticulum stress- and mitochondria-dependent signaling pathways. Environ Toxicol 29(11): 1262-1274, 2014. PMID: 23554011. DOI: 10.1002/ tox. 21857

23 Chen N-C, Chyau C-C, Lee Y-J, Tseng H-C and Chou F-P: Promotion of mitotic catastrophe via activation of pten by paclitaxel with supplement of mulberry water extract in bladder cancer cells. Sci Rep 6: 20417, 2016. PMID: 26838546. DOI: 10.1038/srep20417

$24 \mathrm{Wu}$ JY, Lin SS, Hsu FT and Chung JG: Fluoxetine inhibits DNA repair and nf-kb-modulated metastatic potential in non-small cell lung cancer. Anticancer Res 38(9): 5201-5210, 2018. PMID: 30194168. DOI: 10.21873 /anticanres. 12843

25 Tsai JJ, Pan PJ and Hsu FT: Regorafenib induces extrinsic and intrinsic apoptosis through inhibition of erk/nf-kappab activation in hepatocellular carcinoma cells. Oncol Rep 37(2): 1036-1044, 2017. PMID: 28000898. DOI: 10.3892/or.2016.5328
26 Plati J, Bucur O and Khosravi-Far R: Dysregulation of apoptotic signaling in cancer: Molecular mechanisms and therapeutic opportunities. J Cell Biochem 104(4): 1124-1149, 2008. PMID: 18459149. DOI: $10.1002 /$ jcb. 21707

27 Chen S, Evans HG and Evans DR: FLASH knockdown sensitizes cells to fas-mediated apoptosis via down-regulation of the antiapoptotic proteins, MCL-1 and Cflip short. PLoS One 7(3): e32971, 2012. PMID: 22427918. DOI: 10.1371/journal. pone.0032971

28 Smaili SS, Hsu YT, Sanders KM, Russell JT and Youle RJ: Bax translocation to mitochondria subsequent to a rapid loss of mitochondrial membrane potential. Cell Death Differ 8(9): 909920, 2001. PMID: 11526446. DOI: 10.1038/sj.cdd.4400889

29 Porter AG and Janicke RU: Emerging roles of caspase-3 in apoptosis. Cell Death Differ 6(2): 99-104, 1999. PMID: 10200555. DOI: $10.1038 /$ sj.cdd.4400476

30 Yamana K, Bilim V, Hara N, Kasahara T, Itoi T, Maruyama R, Nishiyama T, Takahashi K and Tomita Y: Prognostic impact of fas/cd95/apo-1 in urothelial cancers: Decreased expression of fas is associated with disease progression. Br J Cancer 93(5): 544551, 2005. PMID: 16091761. DOI: 10.1038/sj.bjc.6602732

31 Gonzalez-Campora R, Davalos-Casanova G, Beato-Moreno A, Garcia-Escudero A, Pareja Megia MJ, Montironi R and LopezBeltran A: Bcl-2, tp53 and bax protein expression in superficial urothelial bladder carcinoma. Cancer Lett 250(2): 292-299, 2007. PMID: 17126995. DOI: 10.1016/j.canlet.2006.10.011

32 Wang J, Zhang X, Wei P, Zhang J, Niu Y, Kang N, Zhang Y, Zhang $\mathrm{W}$ and Xing N: Livin, survivin and caspase 3 as early recurrence markers in non-muscle-invasive bladder cancer. World J Urol 32(6): 1477-1484, 2014. PMID: 24595485. DOI: 10.1007/s00345-014-1246-0

33 Hsu FT, Sun CC, Wu CH, Lee YJ, Chiang CH and Wang WS: Regorafenib induces apoptosis and inhibits metastatic potential of human bladder carcinoma cells. Anticancer Res 37(9): 49194926, 2017. PMID: 28870913. DOI: 10.21873/anticanres.11901

$34 \mathrm{Li} \mathrm{M}$, Song T, Yin ZF and Na YQ: Xiap as a prognostic marker of early recurrence of nonmuscular invasive bladder cancer. Chin Med J (Engl) 120(6): 469-473, 2007. PMID: 17439739.

35 Korkolopoulou P, Goudopoulou A, Voutsinas G, Thomas-Tsagli E, Kapralos P, Patsouris E and Saetta AA: C-flip expression in bladder urothelial carcinomas: Its role in resistance to fas-mediated apoptosis and clinicopathologic correlations. Urology 63(6): 1198-1204, 2004. PMID: 15183989. DOI: 10.1016/ j.urology.2004.01.007

36 Jimenez-Guerrero R, Gasca J and Flores ML: Obatoclax and paclitaxel synergistically induce apoptosis and overcome paclitaxel resistance in urothelial cancer cells. Cancers (Basel) 10(12), 2018. PMID: 30563080. DOI: 10.3390/cancers 10120490

37 Lee S, Yoon CY, Byun SS, Lee E and Lee SE: The role of c-flip in cisplatin resistance of human bladder cancer cells. J Urol 189(6): 2327-2334, 2013. PMID: 23313194. DOI: 10.1016/ j.juro.2013.01.003

38 Bilim V, Kasahara T, Hara N, Takahashi K and Tomita Y: Role of xiap in the malignant phenotype of transitional cell cancer (tcc) and therapeutic activity of xiap antisense oligonucleotides against multidrug-resistant tcc in vitro. Int J Cancer 103(1): 2937, 2003. PMID: 12455050. DOI: 10.1002/ijc.10776

39 Miao C, Liang C, Zhu J, Xu A, Zhao K, Hua Y, Zhang J, Chen W, Suo C, Zhang C, Liu Y, Su S and Wang Z: Prognostic role of matrix metalloproteinases in bladder carcinoma: A systematic review and meta-analysis. Oncotarget 8(19): 32309-32321, 2017. PMID: 28427222. DOI: 10.18632/oncotarget.15907 
40 Maishi N and Hida K: Tumor endothelial cells accelerate tumor metastasis. Cancer Sci 108(10): 1921-1926, 2017. PMID: 28763139. DOI: 10.1111 /cas.13336

41 Shariat SF, Monoski MA, Andrews B, Wheeler TM, Lerner SP and Slawin KM: Association of plasma urokinase-type plasminogen activator and its receptor with clinical outcome in patients undergoing radical cystectomy for transitional cell carcinoma of the bladder. Urology 61(5): 1053-1058, 2003. PMID: 12736046.

42 Vasala K, Paakko P and Turpeenniemi-Hujanen T: Matrix metalloproteinase-2 immunoreactive protein as a prognostic marker in bladder cancer. Urology 62(5): 952-957, 2003. PMID: 14624933
43 Kopparapu PK, Boorjian SA, Robinson BD, Downes M, Gudas LJ, Mongan NP and Persson JL: Expression of vegf and its receptors vegfr1/vegfr2 is associated with invasiveness of bladder cancer. Anticancer Res 33(6): 2381-2390, 2013. PMID: 23749886 .
Received April 27, 2019

Revised May 24, 2019

Accepted May 25, 2019 